\title{
TRIM24 siRNA induced cell apoptosis and reduced cell viability in human nasopharyngeal carcinoma cells
}

\author{
PENG WANG, NA SHEN, DANZHENG LIU, XIANHUI NING, DAQUAN WU and XINSHENG HUANG \\ Department of Otolaryngology, Zhongshan Hospital, Fudan University, Shanghai 200032, P.R. China
}

Received December 16, 2016; Accepted January 4, 2018

DOI: $10.3892 / \mathrm{mmr} .2018 .8946$

\begin{abstract}
Nasopharyngeal carcinoma (NPC) is a common cancer occurring primarily in East Asia and Africa. The high rate of recurrence and metastasis of NPC continuously endangers the health of patients. The present study aimed to identify the underlying mechanisms involved in the progression of NPC and provide experimental basis to develop a novel and efficient agent against NPC. The present study measured the expression level of tripartite motif containing 24 (TRIM24) in tumor tissues from NPC patients using reverse transcription quantitative polymerase chain reaction. Subsequently, Cell Counting kit- 8 and flow cytometry were used to detect the cell proliferation and apoptosis of NPC cell lines HONE1 and CNE1 cells where the TRIM24 gene was knocked-down with small interfering RNA (siRNA). Further, caspase kits and western blot analysis were used to detect the expression of apoptosis and angiogenesis-associated proteins. The present study detected a higher expression level of TRIM24 in tumor tissues and NPC cell lines and lower cell viability and higher apoptotic rate were observed when TRIM24 was silenced. Meanwhile, upregulated caspase- 3 and caspase- 9 indicated induced cell apoptosis in HONE1 and CNE1 cells following the treatment with TRIM24 siRNA. Additionally, the downregulated expression level of vascular endothelial growth factor (VEGF) and VEGF receptor 2 suggested inhibited angiogenesis of NPC cells. Additionally, the reduced levels of janus kinase 2 (JAK2) and signal transducer and activator of transcription 3 (STAT3) indicated a blocked JAK2/STAT3 signaling pathway. However, there was no direct evidence that inactivation of the JAK2/STAT3 signaling pathway was involved in regulation of siTRIM24, these results suggested that TRIM24 has an important role in the growth of NPC. Additionally, silenced TRIM24 may lead to inhibited cell
\end{abstract}

Correspondence to: Mr. Peng Wang, Department of Otolaryngology, Zhongshan Hospital, Fudan University, 180 Fenglin Road, Shanghai 200032, P.R. China

E-mail: wangpengzs78@163.com

*Contributed equally

Key words: nasopharyngeal carcinoma, TRIM24, siRNA proliferation and induced cell apoptosis in NPC cells. The limitation of this study was that HONE1, CNE1 and CNE2 cells may have been contaminated with other cells. Further experiments with validated NPC cells may be needed.

\section{Introduction}

Nasopharyngeal carcinoma (NPC) is the most common cancer occurring in certain regions of East Asia and Africa (1). Over 84,000 new NPC cases are diagnosed annually, with $\sim 80 \%$ occurred in Asia $(2,3)$. All of viral, dietary, genetic and environmental factors contribute to the risk of NPC development $(3,4)$. The main strategies against primary NPC are radiotherapy and comprehensive chemotherapy $(5,6)$. However, the 5-year survival rate was improved to $70 \%$, it is estimated that $15-58 \%$ of patients with NPC experience recurrence of the disease and have to undergo re-treatment $(7,8)$. Local relapse and distant metastasis continue to be a global problem in the treatment of NPC due to the failure to eradicate all tumor cells with conventional treatment. Drug-resistant tumor cells survive after treatment and develop into a new tumor mass or become metastatic (9). Therefore, it is important to develop potential treatments as an efficient and novel therapy against NPC.

The tripartite motif protein, tripartite motif-containing 24 (TRIM24) was identified as a co-regulator of retinoid signaling. It was previously determined to be involved in the progression of several human cancers (10). The aberrant overexpression of TRIM24 may promote tumor development by multiple mechanisms. TRIM24 was reported as a target of chromosomal translocations to form oncogenic fusion proteins in acute promyelocytic leukaemia, papillary thyroid carcinoma and myeloproliferative syndrome $(11,12)$. Additionally, elevated expression of TRIM24 may promote progression of prostate cancer (13) and may be negatively correlated with survival of patients with breast cancer (14). A previous study determined that TRIM24 binds to chromatin and oestrogen receptor to activate oestrogen-dependent genes-associated to cellular proliferation and tumor development (15). Additionally, the expression level of p53 may be negatively regulated by TRIM 24 resulting in the inhibited tumor suppression ability $(16,17)$. However, previous studies have determined the important role of TRIM24 in several human cancers (13-15), and the association between TRIM24 and NPC was not fully reported, which suggested that further investigation in the 
role of TRIM24 in NPC is required in order to determine the possible action mechanisms in tumor development of NPC. The present study detected the expression level of TRIM24 in NPC tissues and evaluated the inhibition ability of TRIM24 small interfering RNA (siRNA) in cell proliferation of NPC cells using NPC cell lines HONE1 and CNE1 transfected with TRIM24 siRNA. Biochemical tests, western blotting and reverse transcription-quantitative polymerase chain reaction (RT-qPCR) were used to determine the expression level of apoptosis, metastasis and signaling pathway-associated proteins in order to determine the role of TRIM24 in tumor development of NPC. The present study aimed to determine the association between TRIM24 with tumor development in NPC and provide the theoretical basis of potential therapies for clinical treatment NPC.

\section{Materials and methods}

Cell culture and transfection. A total of 5 NPC cell lines HONE1 (Shanghai Jining Shiye, Shanghai, China), C666-1 (Shanghai Gaining Biotechnology Co., Ltd., Shanghai, China), CNE2, CNE1 [both from Sai Bai Sheng (Shanghai) Biotechnology Co., Ltd., Shanghai, China] and SUNE-1 [Kelton Biotech (Shanghai) Co., Ltd., Shanghai, China] were cultured in Dulbecco's modified Eagle's medium (DMEM; Hyclone; GE Healthcare Life Sciences, Logan, UT, USA) containing $10 \%$ fetal calf serum (Gibco; Invitrogen; Thermo Fisher Scientific, Inc., Waltham, MA, USA) and $1 \% 100 \mathrm{x}$ mycillin (Beijing Solarbio Science \& Technology Co., Ltd., Beijing, China) with $5 \% \mathrm{CO}_{2}$ at $37^{\circ} \mathrm{C}$. NPC cell lines with high expression level of TRIM 24 were determined by using RT-qPCR analysis. Cell viability of selected cell lines (HONE1 and CNE1) was detected at 95\% using Trypan blue staining for $5 \mathrm{~min}$ at room temperature. Then the cells were digested and seeded into 6 -well plate $\left(5 \times 10^{5}\right.$ cells/well) prior to transfection. Lipofectamine 2000 (Invitrogen; Thermo Fisher Scientific, Inc.) was used to transfect $5 \mu 1$ TRIM2 4 siRNA (Shanghai GenePharma Co., Ltd., Shanghai, China) or negative control siRNA (Shanghai GenePharma Co., Ltd.) into HONE1 and CNE1 cell lines. After incubation for $48 \mathrm{~h}$, the transfected cells were collected and processed for cell viability, apoptosis, biochemical tests, RT-qPCR and western blot assay.

Western blot assay. Western blot assay was used to determine the expression levels of TRIM24 and associated proteins in NPC cell lines. Cultured or transfected cells were washed with 1xPBS twice, followed by the radioimmunoprecipitation assay lysis buffer (Beijing Solarbio Science \& Technology Co., Ltd.) at $4^{\circ} \mathrm{C}$. Following lysis, samples were centrifuged at $12,000 \mathrm{x}$ g for $15 \mathrm{~min}$ at $4^{\circ} \mathrm{C}$ and the supernatant was collected. Proteins were quantified by using the bicinchoninic acid assay. Protein $(15 \mu \mathrm{l})$ with loading buffer were heated in a boiling water bath for $10 \mathrm{~min}$ and then centrifuged at $12,000 \mathrm{x} \mathrm{g}$ for $10 \mathrm{~min}$ at room temperature. The supernatant (30 $\mu \mathrm{g}$ protein) was collected and loaded on 10\% SDS-polyacrylamide gels. Following electrophoresis, proteins on the gel were transferred onto a nitrocellulose blotting membrane (EMD Millipore, Billerica, MA, USA) and incubated with 5\% non-fat milk at $4^{\circ} \mathrm{C}$ overnight. The membrane was washed and incubated with TRIM24 (Abcam, Cambridge, UK; cat. no. Ab174287;
1:1,000), vascular endothelial growth factor (VEGF; Abcam; cat. no. Ab46154; 1:1,000), VEGF receptor 2 (VEGFR2; Affinity, Cincinnati, OH, USA; cat. no. AF6281; 1:1,000), caspase-3 (Abcam; cat. no. Ab44976; 1:500), caspase-9 (Abcam; cat. no. Ab2013; 1:1,000), janus kinase 2 (JAK2; Cell Signaling Technology, Inc., Danvers, MA, USA; cat. no. 3230; 1:1,000), phosphorylated (p)-JAK2 (Cell Signaling Technology, Inc.; cat. no. 8082; 1:1,000), signal transducer and activator of transcription 3 (STAT3; Abcam; cat. no. Ab76315; 1:2,000), p-STAT3 (cat. no. 9139; 1:1,500) and GAPDH (both Cell Signaling Technology, Inc.; cat. no. 5174; 1:2,000) antibodies for $2 \mathrm{~h}$ at room temperature. Then the membrane was washed with TBS- $0.05 \%$ Tween-20 and incubated with anti-horseradish peroxidase secondary antibody (Beyotime Institute of Biotechnology, Shanghai, China; cat. nos. A0208 and A0216; 1:1,000 dilution) for $1 \mathrm{~h}$. Immunoreactive bands were detected using an enhanced chemiluminescence detection kit and an LAS-4000 mini system (Fujifilm Corporation, Kumamoto, Japan) was used for visualization. Western blotting was repeated three times. The films of western blotting were then scanned by using a Bio-Rad imaging densitometer (ModelGS-700; Bio-Rad Laboratories, Inc., Hercules, CA, USA) and the densities of the bands were semi-quantified using Quantity One software Version 4.62 (Bio-Rad Laboratories, Inc.).

RT-qPCR analysis. PCR analysis was preformed to determine the expression level of TRIM24 in NPC tissue samples and associated proteins in NPC cell lines. The gene expression of TRIM24 in 35 tissue samples with para-carcinoma tissues were determined using RT-qPCR. Paired human NPC tissues and para-carcinoma tissues were collected from 35 patients (including 23 males and 12 females, age range: 31 to 74 years) that underwent standard surgical procedures between January 2014 and December 2014 in Department of Otolaryngology, Zhongshan Hospital (Shanghai, China) after the written informed consent of the patients was obtained. The experimental protocols were approved by the Institutional Review Committees of Zhongshan Hospital (Shanghai, China). Tissue samples were collected from patients with complete clinical and pathological follow-up data.

Total RNA was extracted from tissue samples by using TRIzol reagent (Invitrogen; Thermo Fisher Scientific, Inc.). Reverse transcription kit (Fermentas; Thermo Fisher Scientific, Inc.) was used to syntheses cDNA through a total volume of $25 \mu \mathrm{l}$ (12 $\mu \mathrm{l}$ RNA-primer mix, $5 \mu \mathrm{l}$ 5XRT reaction buffer, $1 \mu \mathrm{l} 25 \mathrm{mM}$ dNTPs, $1 \mu 125 \mathrm{U} / \mu 1$ RNase inhibitor, $1 \mu 1$ $200 \mathrm{U} / \mu \mathrm{l}$ M-MLV reverse transcriptase, $1 \mu \mathrm{l}$ Oligo(dt) $)_{18}$ and $4 \mu \mathrm{l}$ DNase-free $\mathrm{ddH}_{2} \mathrm{O}$ ) and the thermocycling conditions were: $37^{\circ} \mathrm{C}$ for $60 \mathrm{~min}, 85^{\circ} \mathrm{C}$ for $5 \mathrm{~min}$ and $4^{\circ} \mathrm{C}$ for $5 \mathrm{~min}$ for the RT step. Then cDNA was amplified by using SYBR-Green PCR kit (Thermo Fisher Scientific, Inc.) using a total PCR system of $25 \mu \mathrm{l}$ (12.5 $\mu \mathrm{l}$ SYBR-Green Mix, $0.5 \mu \mathrm{l}$ forward

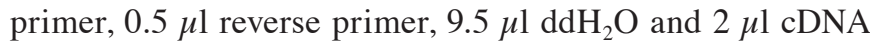
template) with the PCR thermocycling conditions were as follows: $10 \mathrm{~min}$ at $95^{\circ} \mathrm{C}$, and then $15 \mathrm{sec}$ at $95^{\circ} \mathrm{C}$ and $45 \mathrm{sec}$ at $60^{\circ} \mathrm{C}$ for 40 cycles. Amplification kinetic curves were obtained through $15 \mathrm{sec}$ at $95^{\circ} \mathrm{C}, 1 \mathrm{~min}$ at $60^{\circ} \mathrm{C}, 15 \mathrm{sec}$ at $95^{\circ} \mathrm{C}$ and $15 \mathrm{sec}$ at $60^{\circ} \mathrm{C}$. Data was collected by using ABI Prism 7300 SDS software (Applied Biosystem, Thermo Fisher Scientific, 
Table I. Primers for quantitative polymerase chain reaction analysis.

\begin{tabular}{lllc}
\hline Gene & \multicolumn{1}{c}{ Forward (5'-3') } & \multicolumn{1}{c}{ Reverse (5'-3') } & Amplicon size (bp) \\
\hline TRIM24 & CCAGCCAAGACCACCCTCAAAC & CAGAGCTTCCTCGGCTTCCAAC & 149 \\
VEGF & ATTTCTGGGATTCCTGTAG & CAGTGAAGACACCAATAAC & 157 \\
VEGFR2 & CTCAGCAGGATGGCAAAG & ACTGTCCGTCTGGTTGTC & 278 \\
Caspase-3 & AACTGGACTGTGGCATTGAG & ACAAAGCGACTGGATGAACC & 161 \\
Caspase-9 & CCTCACCCTGCCTTATCTTG & TCCCTCTTCCTCCACTGTTC & 189 \\
GAPDH & AATCCCATCACCATCTTC & AGGCTGTTGTCATACTTC & 218 \\
\hline
\end{tabular}

TRIM24, tripartite motif containing 24; VEGF, vascular endothelial growth factor; VEGFR2, VEGF receptor 2.

Inc.) and quantified as previously described (18). Primers used for the amplification were listed in Table I.

Cell Counting kit-8 (CCK-8) assay. Cultured HONE1 and CNE1 cells were trypsinized and diluted to $2 \times 10^{4}$ cells $/ \mathrm{ml}$. Then cells were plated into 96 -well plates $\left(2 \times 10^{3}\right.$ cells/well $)$ and incubated at $37^{\circ} \mathrm{C}$ for $12 \mathrm{~h}$, followed by the transfection with TRIM24 siRNA or negative control siRNA, respectively. Transfected cells in each well were mixed with $100 \mu 1$ DMEM containing 10\% CCK-8 (Dojindo Molecular Technologies, Inc., Kumamoto, Japan) at 0, 24, 48 and $72 \mathrm{~h}$, and then incubated in $5 \% \mathrm{CO}_{2}$ at $37^{\circ} \mathrm{C}$ for $1 \mathrm{~h}$. The optical density (OD) $450 \mathrm{~nm}$ of cell suspension was measured using a spectrophotometer in order to evaluate the cell viability of HONE1 and CNE1 cells.

Flow cytometry. Transfected cells were formed into a single cell suspension by $0.25 \%$ trypsin and washed by $10 \% \mathrm{PBS}$, followed by the centrifugation at $1,000 \mathrm{x}$ g for $5 \mathrm{~min}$ at $4^{\circ} \mathrm{C}$. Supernatant was discarded and then the cells were incubated with Annexin-V fluorescein isothiocyanate (FITC) apoptosis detection kit (BD Biosciences, Franklin Lakes, NJ, USA) for $10 \mathrm{~min}$ at room temperature without light. Cell apoptosis rate was measured by using flow cytometry (FACS Calibur, BD Biosciences) and FlowJo Software version 7.6 (FlowJo LLC, Ashland, OR, USA).

Biochemical test. Caspase-3 and caspase-9 concentrations were assessed using Caspase-3 and caspase-9 colorimetric assay kits (KeyGen Biotech Co., Ltd., Shanghai, China). Cultured and transfected cells were collected and washed by using PBS for twice, followed with lysis with $100 \mu$ l pre-cooled lysis buffer containing $1 \mu \mathrm{l}$ dithiothreitol (DTT). Lysed cells were centrifuged at $12,000 \mathrm{x}$ g for $1 \mathrm{~min}$. Supernatant was collected and protein concentrations were determined using the Bradford method. $50 \mu \mathrm{l}$ supernatant was mixed with $50 \mu \mathrm{l}$ 2X Reaction Buffer containing 0.5 $\mu 1$ DTT and $5 \mu$ l Caspase-3 or Caspase-9 substrate, respectively. After $4 \mathrm{~h}$ of incubation at $37^{\circ} \mathrm{C}$, the concentrations of Caspase- 3 and Caspase- 9 were measured at OD $400 \mathrm{~nm}$.

Statistical analysis. All the experiments were repeated three times and the values are expressed as the mean \pm standard deviation. Statistical comparisons were performed using one-way analysis of variance followed by the Tukey post hoc test with GraphPad Prism software (version 6.0; Graphpad
Software, Inc., San Diego, CA, USA). P $<0.05$ was considered to indicate a statistically significant difference.

\section{Results}

Screening and identification of nasopharyngeal cancer cell lines. As presented in Fig. 1A, the increased expression level of TRIM24 measured by RT-PCR in NPC tissues demonstrated a significant difference compared with the paracancerous tissues, indicating the overexpression of TRIM24 in NPC tissues and suggesting an association between TRIM24 and NPC. To further investigate the association between TIRM24 and NPC, NPC cell lines HONE1 and CNE1 with higher expression levels of TRIM24, as determined using western blotting, were selected for further experiments (Fig. 1B and C).

Furthermore, western blotting and qPCR were used to identify the interference effect of TRIM24 siRNA on the expression of TRIM24 in HONE1 and CNE1 cells. As presented in Fig. 1D and E, the expression level of TRIM24 declined significantly in HONE1 and CNE1 cells $(\mathrm{P}<0.05$ or $\mathrm{P}<0.001)$. The gene expression of TRIM24 was additionally decreased, with a significant difference compared with the negative control group (Fig. 1F; $\mathrm{P}<0.001$ ), indicating the efficient gene silencing of TRIM24 siRNA.

TRIM24 siRNA inhibits cell proliferation. As presented in Fig. 2, the cell viability of HONE1 and CNE1 cells detected via CCK8 assay was decreased following treatment with TRIM24 siRNA, in a time-dependent manner. The decreased cell viability was significant compared with the control and negative control groups, indicating the inhibitory effect of TRIM24 siRNA on cell proliferation in HONE1 and CNE1 cells.

TRIM24 siRNA induces cellular apoptosis. The results of the flow cytometry demonstrated cellular apoptosis in HONE1 and CNE1 cells induced by TRIM24 siRNA (Fig. 3). The percentages of early apoptotic cells presented in the lower right quadrant of the histograms indicated the induced cellular apoptosis in the TRIM24 siRNA group, suggesting an apoptosis-promoting effect of TRIM24 siRNA on HONE1 and CNE1 cells. Furthermore, the expression of the pro-apoptotic proteins caspase- 3 and caspase- 9 , measured using biochemical analysis, exhibited an upregulation following treatment with TRIM24 siRNA, which corresponded to the results of the flow cytometry (Fig. 3B and D). Additionally, the upregulated 


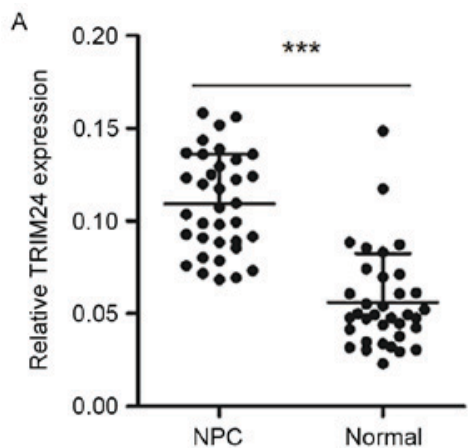

C
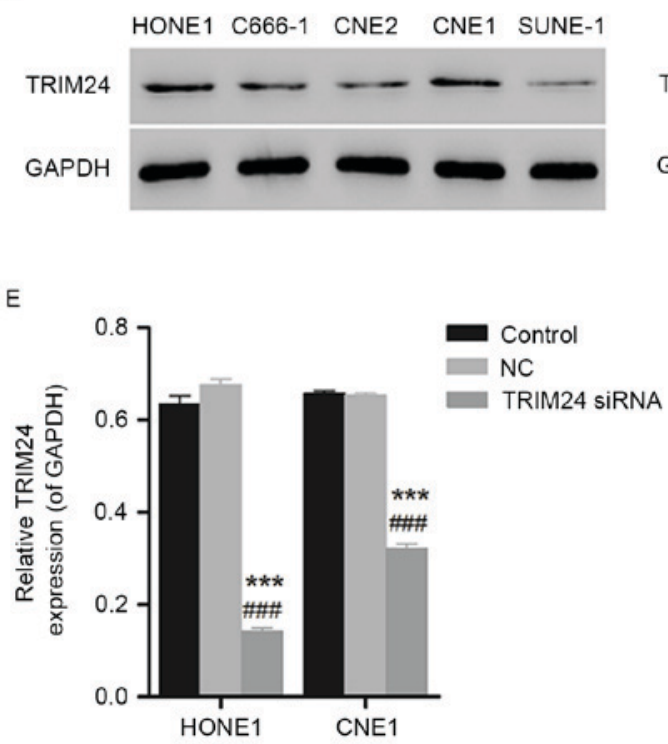

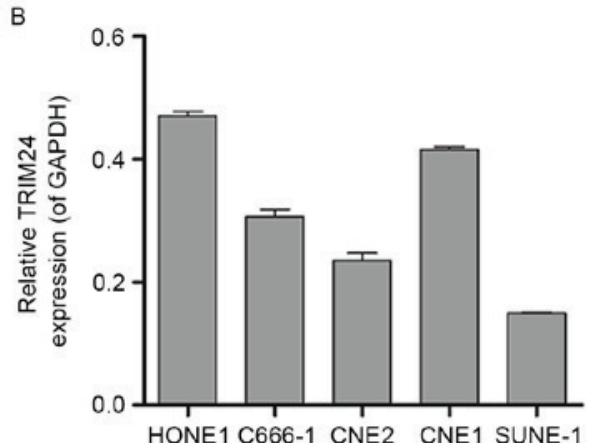

D

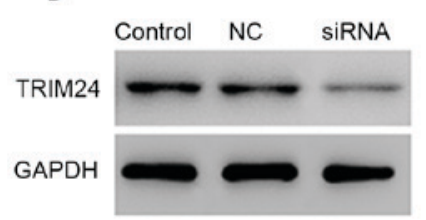

HONE1

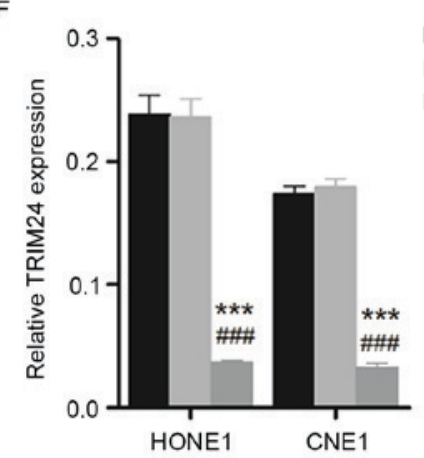

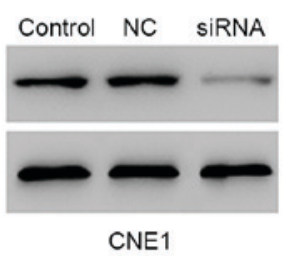

Control
NC
TRIM24 siRNA

Figure 1. Detection of TRIM24 expression in NPC tissues, NPC cell lines and cells transfected with TRIM24 siRNA. (A) qPCR was used to measure the gene expression level of TRIM24 in 35 tissue samples with para-carcinoma tissues. A higher expression level of TRIM24 was detected in NPC tissues. ${ }^{* * *} \mathrm{P}<0.001$ vs. normal tissues. The expression level of TRIM24 in human NPC cell lines including HONE1, C666-1, CNE2, CNE1 and SUNE-1 was (B) quantified following detection using (C) western blotting. (D) Western blotting and (E) quantification was used to measure the TRIM24 expression in HONE1 and CNE1 cells following transfection with TRIM24 siRNA. ${ }^{* * * *} \mathrm{P}<0.001$ vs. control group. ${ }^{* \# \#} \mathrm{P}<0.001$ vs. NC group $(\mathrm{n}=3)$. (F) The gene expression level of TRIM24 was also measured using qPCR. ${ }^{* * *} \mathrm{P}<0.001$ vs. control group. ${ }^{\# \# \#} \mathrm{P}<0.001$ vs. NC group $(\mathrm{n}=3)$. TRIM24, tripartite motif containing 24 ; NPC, nasopharyngeal carcinoma; NC, negative control; siRNA, small interfering RNA; qPCR, quantitative polymerase chain reaction.
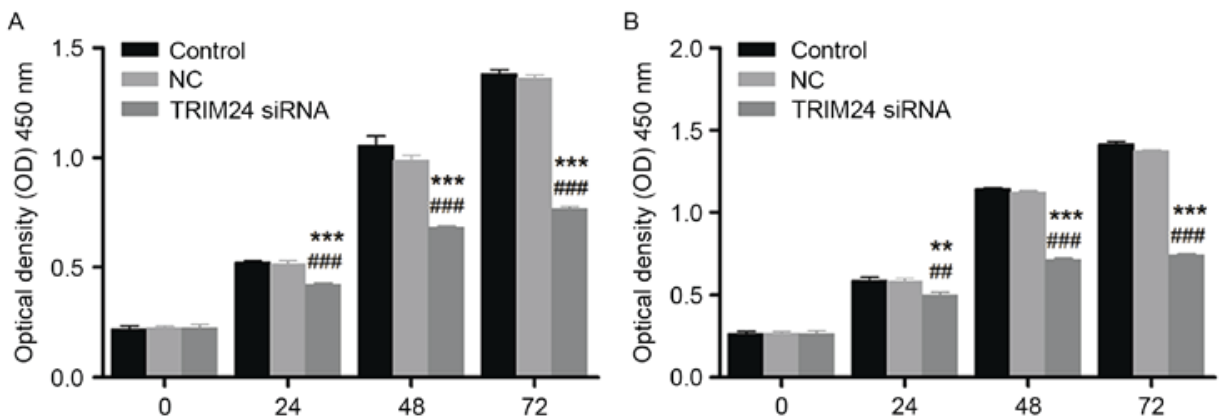

Figure 2. TRIM24 siRNA reduces the cell viability of nasopharyngeal carcinoma cell lines HONE1 and CNE1. (A) The cell viability of HONE1 cells was determined using a CCK-8 assay following transfection with TRIM24 siRNA for $0,24,48$ and $72 \mathrm{~h} .{ }^{* * * *} \mathrm{P}<0.001$ vs. control group. ${ }^{\# \# \#} \mathrm{P}<0.001$ vs. NC group $(\mathrm{n}=3)$. (B) The inhibition effect of TRIM24 siRNA on the proliferation of CNE1 cells was also evaluated using a CCK-8 assay. ${ }^{* * *} \mathrm{P}<0.01,{ }^{* * * * *} \mathrm{P}<0.001$ vs. control group. ${ }^{\# \#} \mathrm{P}<0.01,{ }^{\# \# /} \mathrm{P}<0.001$ vs. NC group $(\mathrm{n}=3)$. TRIM24, tripartite motif containing 24; siRNA, small interfering RNA; NC, negative control; CCK-8, Cell Counting kit-8.

caspase-3 and caspase-9 measured by western blotting and qPCR indicated the pro-apoptotic ability of TRIM24 siRNA on HONE1 and CNE1 cells, suggesting an important role for TRIM24 in NPC (Fig. 3E-G).
TRIM24 siRNA blocks the JAK2/STAT3 signaling pathway. To further investigate the possible mechanisms involved in inhibited cellular apoptosis and cell proliferation due to TRIM24 siRNA, the expression levels of the cellular 

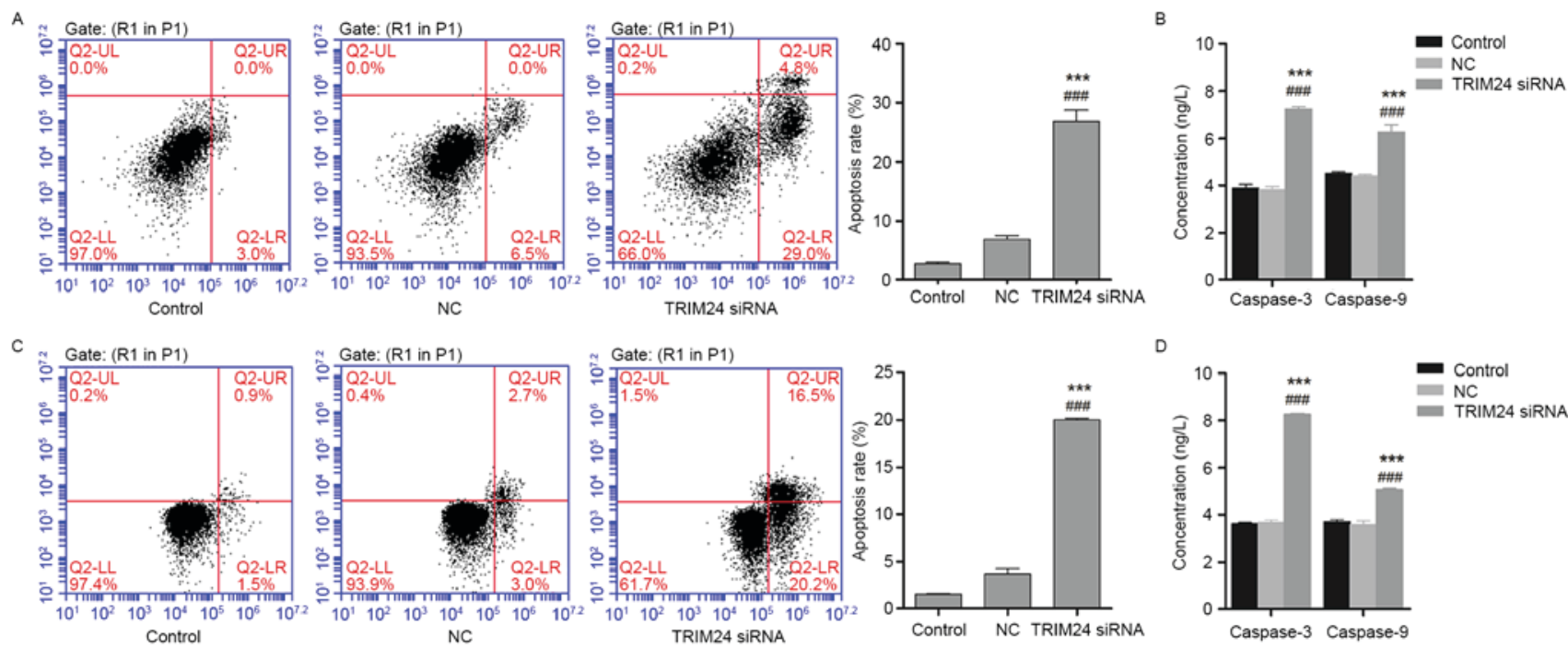

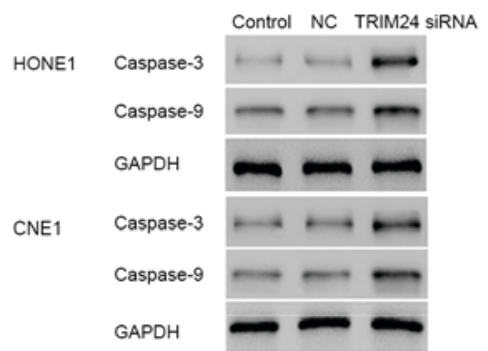

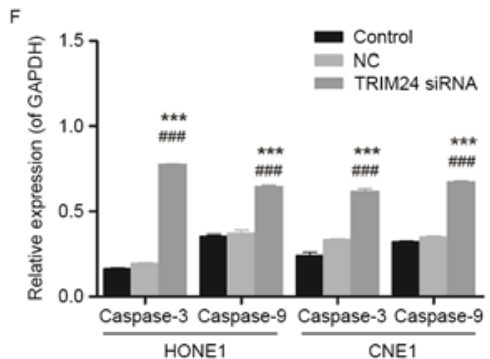

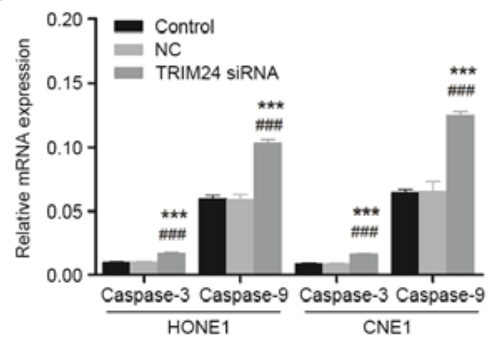

Figure 3. TRIM24 siRNA induces cellular apoptosis in HONE1 and CNE1 cells. (A) Cellular apoptosis in HONE1 cells was evaluated using flow cytometry following transfection with TRIM24 siRNA for $48 \mathrm{~h}$. Early apoptotic cells were identified using Annexin V/propidium iodide double staining and are exhibited in the lower right quadrant. ${ }^{* * *} \mathrm{P}<0.001$ vs. control group. ${ }^{\# \#} \mathrm{P}<0.001$ vs. NC group $(\mathrm{n}=3)$. (B) Apoptosis-associated proteins caspase-3 and caspase-9 were quantified. ${ }^{* * *} \mathrm{P}<0.001$ vs. the control group. ${ }^{\# \# *} \mathrm{P}<0.001$ vs. the $\mathrm{NC}$ group $(\mathrm{n}=3)$. (C) TRIM24 siRNA treatment induced cell apoptosis and (D) increased caspase proteins were also identified in CNE1 cells. ${ }^{* * *} \mathrm{P}<0.001$ vs. control group. ${ }^{\# \# \#} \mathrm{P}<0.001$ vs. NC group $(\mathrm{n}=3)$. HONE1 and CNE1 cells where TRIM24 was silenced had upregulated caspase-3 and caspase-9 levels, according to the (E) western blotting and (F) quantification. (G) HONE1 and CNE1 cells transfected with TRIM24 siRNA exhibited increased caspase-3 and caspase-9 mRNA expression, corresponding to the results obtained in western blot analysis. ${ }^{* * * *} \mathrm{P}<0.001$ vs. control group. ${ }^{\# \# \#} \mathrm{P}<0.001$ vs. $\mathrm{NC}$ group $(\mathrm{n}=3)$. TRIM24, tripartite motif containing 24; siRNA, small interfering RNA; NC, negative control.

apoptosis- and tumorigenesis-associated proteins caspase-3, caspase-9, VEGF and VEGFR2 in HONE1 and CNE1 cells was measured by western blotting and qPCR. Compared with the control group, the expression levels of VEGF and VEGFR2 exhibited a downregulation with a significant difference in HONE1 and CNE1 cells transfected with TRIM24 siRNA (Fig. 4A and B; $n=3 ; \mathrm{P}<0.001$ ). Similar results were observed with the PCR analysis (Fig. 4C). Besides, the expression levels of the pro-apoptotic proteins caspase- 3 and caspase- 9 exhibited an upregulation, corresponding to the results of the flow cytometry. In addition, the expression of the JAK2/STAT3 signaling pathway-associated proteins JAK2 and STAT3 was measured using western blotting and qPCR. As presented in Fig. 4A, D and E, decreased expression of JAK 2 and STAT3 indicated the inhibition of the JAK2/STAT3 signaling pathway. However, further evidence is required to demonstrate the direct action of siTRIM24 on the inactivation of JAK2/STAT3 signaling pathway.

\section{Discussion}

TRIM24 belongs to the tripartite motif superfamily and was initially identified as a fusion partner of the B-raf protein in the oncoprotein T18, observed in mouse hepatocellular carcinoma (19). TRIM24 has been reported to be overexpressed in a number of types of cancer. Upregulated TRIM24 was observed in acute promyelocytic leukaemia, myeloproliferative syndrome, papillary thyroid carcinoma, breast cancer and non-small cell lung cancer $(11,12,19,20)$, which indicated that TRIM24 may serve an oncogenic role in carcinogenesis. In addition, overexpression of TRIM24 is associated with survival in breast cancer patients (14), and with pTNM stage and differentiation in non-small cell lung cancer (20). These previous studies suggested that the TRIM24 gene may serve an important role in tumorigenesis. In the present study, it was demonstrated that the expression of TRIM24 in NPC tissues was increased compared with noncancerous tissues. These findings suggested that TRIM24 may serve an important role in the progression of human NPC.

In order to confirm the potential role of TRIM24 in human NPC development, the present study employed siRNA to knock down TRIM24 in HONE1 and CNE1 cell lines for further study. A CCK-8 assay was used to investigate the role of TRIM24 in cell proliferation. The cell proliferation of HONE1 and CNE1 cells following TRIM24 silencing was downregulated compared with the control group, which suggested that TRIM24 may promote cell proliferation in HONE1 and CNE1 cells. Subsequently, cell apoptosis of HONE1 and CNE1 cells with TRIM24 knockdown, and the 

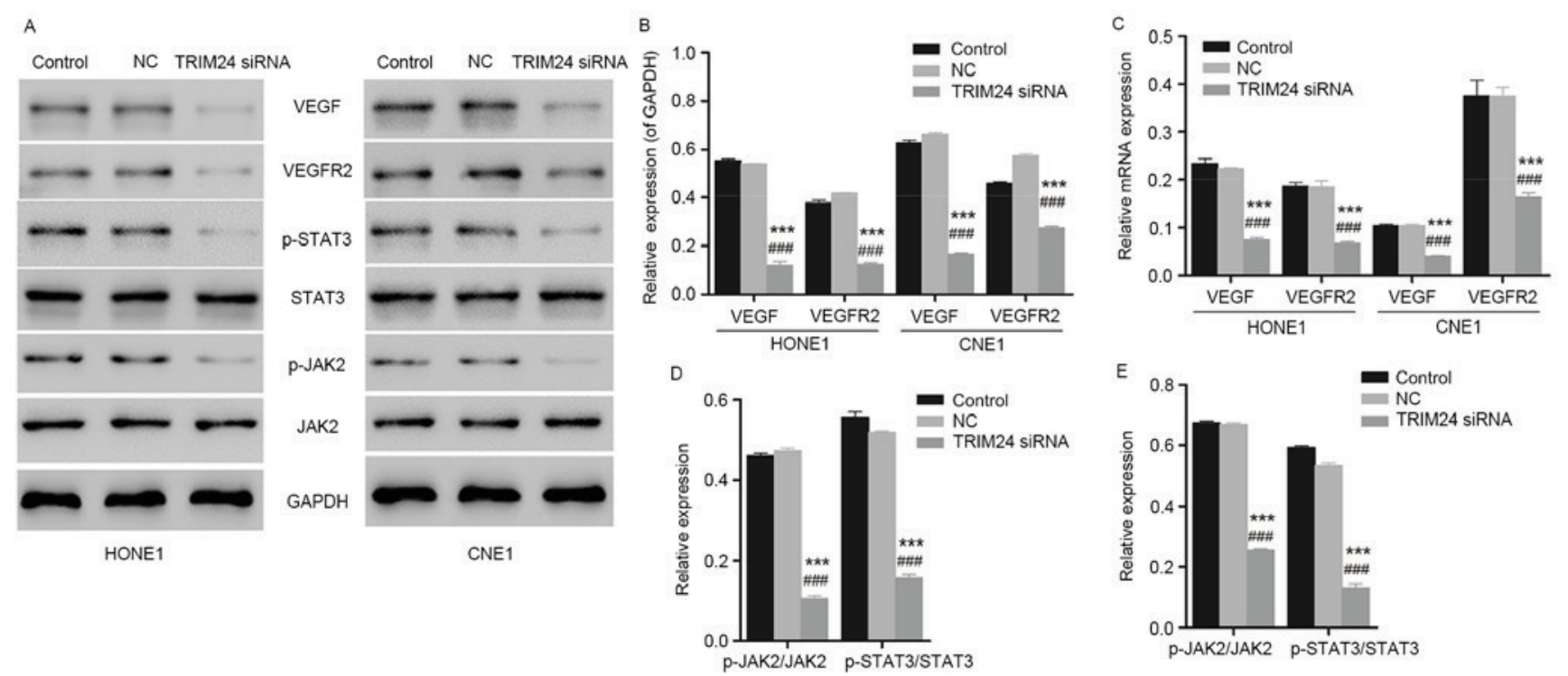

Figure 4. Expression level of VEGF, VEGFR2, JAK2 and STAT3 in HONE1 and CNE1 cells, determined using western blotting and qPCR. (A) Detection and visualization of immunoreactive bands via western blot analysis. (B) HONE1 and CNE1 cells with TRIM24 silenced had downregulated protein expression of VEGF and VEGFR2, indicating the inhibited angiogenesis. ${ }^{* * *} \mathrm{P}<0.001$ vs. control group. ${ }^{\# \#} \mathrm{P}<0.001$ vs. NC group (n=3). (C) HONE1 and CNE1 cells transfected with TRIM24 siRNA exhibited reduced mRNA expression of VEGF and VEGFR2, corresponding to the results obtained in the western blot analysis. ${ }^{* * *} \mathrm{P}<0.001$ vs. control group. ${ }^{\# \#} \mathrm{P}<0.001$ vs. NC group (n=3). (D) HONE1 cells with TRIM24 silenced presented downregulated JAK2 and STAT3, indicating the blocked JAK2/STAT3 signaling pathway. ${ }^{* * *} \mathrm{P}<0.001$ vs. control group. ${ }^{\# \#} \mathrm{P}<0.001$ vs. NC group (n=3). (E) Similar results were obtained in CNE1 cells. ${ }^{* * *} \mathrm{P}<0.001$ vs. control group. ${ }^{\# \# \#} \mathrm{P}<0.001$ vs. $\mathrm{NC}$ group $(\mathrm{n}=3)$. NC, negative control; TRIM24, tripartite motif containing 24 ; siRNA, small interfering RNA; VEGF, vascular endothelial growth factor; VEGFR2, VEGF receptor 2; STAT3, signal transducer and activator of transcription 3; p-STAT3, phosphorylated-STAT3; JAK2, janus kinase 2; p-JAK2, phosphorylated-JAK2.

apoptosis-associated proteins caspase-3 and caspase-9, were investigated using an Annexin/V kit and biochemical assays. Caspase-3 activated by apoptotic signals acts on peptide chains and splits substrates, resulting in the apoptosis of cells $(21,22)$. Caspase- 3 serves a direct role in cellular apoptosis as one of the effector caspases. It was previously reported that caspase- 3 induced the activation of caspase-activated deoxyribonuclease (CAD) in the degradation of DNA. When apoptosis occurs, CAD with nuclease activity is released by its inhibitor I ${ }^{\mathrm{CAD}}$, activated by caspase-3 (23). The nuclear lamina, which is responsible for the stability of chromatin, may be cut by caspase- 3 at a single site, resulting in the degradation of chromatin (23). The results of the flow cytometry analysis performed in the present study demonstrated a significant increase in the population of apoptotic HONE1 cells following treatment with TRIM24 siRNA compared with the negative control, in addition to CNE1 cells. Biochemical and western blot analyses demonstrated that the depletion of TRIM24 upregulated caspase-3 and caspase- 9 expression. These results suggested that TRIM24 may suppress cellular apoptosis and differentiation via caspase-3 and caspase- 9 . The above results may explain the mechanism of TRIM24 in human NPC cell differentiation.

One of the most intractable characteristics of NPC is metastasis (24). Tumor angiogenesis factors involved in migration serve an important role in the generation of tumor vessels, resulting in the uncontrolled growth of the tumor (25). The process of angiogenesis, including the proliferation and migration of endothelial cells, the degradation of the basement membrane and the formation of the cavity, is a complex process regulated by a number of positive and negative regulatory factors (25). VEGF is a type of specific heparin-binding growth factor in vascular endothelial cells. VEGF, VEGFA and VEGFB have been demonstrated to induce angiogenesis through the recruitment of tumor-associated macrophages, which serve an important role in the angiogenesis and growth of tumor cells in gastric cancer (26). The association between the high expression of VEGF and metastasis, angiogenesis and survival in rectal cancer has additionally been reported (27). According to the results obtained in the western blot and PCR analyses in the present study, downregulated expression levels of VEGF and VEGFR2, in HONE1 and CNE1 cells following treatment with TRIM24 siRNA, suggested that silenced TRIM24 decreased angiogenesis and the metastatic ability of NPC cells significantly compared with the negative control. Furthermore, these results indicated an association between TRIM24, and a higher incidence of metastasis and recurrence in NPC.

The JAK2/STAS3 signaling pathway, with a range of functions including proliferation, differentiation, apoptosis, cell cycle and immunoregulation, is a signal transduction pathway stimulated by cytokines $(28,29)$. JAK2 is a type of non-transmembrane tyrosine kinase. STAT3 serves an important role in signal transduction and transcriptional activation. Constitutive activation of JAK2 has been reported in childhood T cell acute lymphoblastic leukemia (30). STAT3 has been observed to be constitutively activated in breast carcinoma and non-small cell lung cancer, correlated with induced cell proliferation and inhibited cellular apoptosis (31). It has been reported that suppression of the JAK2/STAT3 signaling pathway inhibited cell growth and induced cellular apoptosis in various cancer cells (32). Previous studies revealed suppressed cell growth, induced cellular apoptosis 
and arrest of the cell cycle in colorectal cancer cells due to blocked JAK2/STAT3 signaling (33). In the present study, decreased JAK 2 and STAT3 expression levels indicated the inhibition of the JAK2/STAS3 signaling pathway in HONE1 and CNE1 cells, resulting in the inhibited cell proliferation and induced cellular apoptosis. However, there is no direct evidence indicating the association between the regulation of siTRIM24 and the inhibition of the JAK2/STAT3 signaling pathway at present. Overexpression of TRIM24 was identified in tumor growth due to the activation of the upstream extracellular signal-regulated kinase signaling pathway (34), while the interaction between TRIM24 and JAK2/STAT3 remains unclear. It was hypothesized that the inhibition of the JAK2/STAT3 signaling pathway may be involved in the regulation of TRIM24. Further studies are required to investigate the possible mechanisms, including the functional channels involved.

In conclusion, the present demonstrated the overexpression of TRIM24 in human NPC and the important role of TRIM24 in the growth of NPC cells. Furthermore, silencing of TRIM24 may cause inhibited cell proliferation and induced cellular apoptosis in NPC cells. The limitation of the present study was that HONE 1, CNE1 and CNE2 cells may have been contaminated with HeLa cells or cells of unknown origin. Further experiments with validated NPC cells may be needed.

\section{Acknowledgements}

Not applicable.

\section{Funding}

No funding was received.

\section{Availability of data and materials}

The analyzed data sets generated during the study are available from the corresponding author on reasonable request.

\section{Authors' contributions}

PW conceived and designed the study. PW, NS, DL, XN, DW and $\mathrm{XH}$ performed the experiments. PW and NS wrote the manuscript. All authors read and approved the manuscript.

\section{Ethics approval and consent to participate}

The experimental protocols were approved by the Institutional Review Committees of ZhongShan Hospital (Shanghai, China). Written informed consent of the patients was obtained.

\section{Consent for publication}

Written informed consent of the patients was obtained.

\section{Competing interests}

The authors declare they have no competing interests.

\section{References}

1. Lee AW, Ng W, Chan Y, Sze H, Chan C and Lam T: The battle against nasopharyngeal cancer. Radiother Oncol 104: 272-278, 2012.

2. Qu S, Liang ZG and Zhu XD: Advances and challenges in intensity-modulated radiotherapy for nasopharyngeal carcinoma. Asian Pac J Cancer Prev 16: 1687-1692, 2015.

3. Nor Hashim NA, Ramzi NH, Velapasamy S, Alex L, Chahil JK, Lye SH, Munretnam K, Haron MR and Ler LW: Identification of genetic and non-genetic risk factors for nasopharyngeal carcinoma in a southeast asian population. Asian Pac J Cancer Prev 13: 6005-6010, 2012.

4. Li JX, Lu TX, Huang Y and Han F: Clinical characteristics of recurrent nasopharyngeal carcinoma in high-incidence area. ScientificWorldJournal 2012: 719754, 2012.

5. Chau RM, Teo PM, Kam MK, Leung S, Cheung K and Chan AT: Dosimetric comparison between 2-dimensional radiation therapy and intensity modulated radiation therapy in treatment of advanced T-stage nasopharyngeal carcinoma: To treat less or more in the planning organ-at-risk volume of the brainstem and spinal cord. Med Dosim 32: 263-270, 2007.

6. Chen AM, Yang C, Marsano J, Liu T and Purdy J: Intensity-modulated radiotherapy for nasopharyngeal carcinoma: Improvement of the therapeutic ratio with helical tomotherapy vs segmental multileaf collimator-based techniques. Br J Radiol 85: e537-e543, 2012.

7. Du L, Zhang XX, Ma L, Feng LC, Li F, Zhou GX, Qu BL, Xu SP, Xie CB and Yang J: Clinical study of nasopharyngeal carcinoma treated by helical tomotherapy in China: 5-year outcomes. Biomed Res Int 2014: 980767, 2014.

8. FangFM,Chien CY,TsaiWL,ChenHC,HsuHC,LuiCC,HuangTL and Huang HY: Quality of life and survival outcome for patients with nasopharyngeal carcinoma receiving three-dimensional conformal radiotherapy vs. intensity-modulated radiotherapy-a longitudinal study. Int J Radiat Oncol Biol Phys 72: 356-364, 2008.

9. He X, Ye M, Guo X, Pan Z, Zhang Z, He S and Liu T: Treatment outcome of patients with stages I-II nasopharyngeal carcinoma after late course accelerated hyperfractionation radiotherapy alone. Oral Oncol 48: 1058-1063, 2012.

10. Hatakeyama S: TRIM proteins and cancer. Nat Rev Cancer 11: 792-804, 2011

11. Belloni E, Trubia M, Gasparini P, Micucci C, Tapinassi C, Confalonieri S, Nuciforo P, Martino B, Lo-Coco F, Pelicci PG and Di Fiore PP: 8p11 myeloproliferative syndrome with a novel $\mathrm{t}(7 ; 8)$ translocation leading to fusion of the FGFR1 and TIF1 genes. Genes Chromosomes Cancer 42: 320-325, 2005.

12. Zhong S, Delva L, Rachez C, Cenciarelli C, Gandini D, Zhang H, Kalantry S, Freedman LP and Pandolfi PP: A RA-dependent, tumour-growth suppressive transcription complex is the target of the PML-RAR $\alpha$ and T18 oncoproteins. Nat Genet 23: 287-295, 1999.

13. Groner AC, Cato L, de Tribolet-Hardy J, Bernasocchi T, Janouskova H, Melchers D, Houtman R, Cato ACB, Tschopp P, $\mathrm{Gu}$ L, et al: TRIM24 is an oncogenic transcriptional activator in prostate cancer. Cancer Cell 29: 846-858, 2016.

14. Chambon M, Orsetti B, Berthe ML, Bascoul-Mollevi C, Rodriguez C, Duong V, Gleizes M, Thénot S, Bibeau F, Theillet C and Cavaillès V: Prognostic significance of TRIM24/TIF-1 $\alpha$ gene expression in breast cancer. Am J Pathol 178: 1461-1469, 2011.

15. Tsai WW, Wang Z, Yiu TT, Akdemir KC, Xia W, Winter S, Tsai CY, Shi X, Schwarzer D, Plunkett W, et al: TRIM24 links a non-canonical histone signature to breast cancer. Nature 468: 927-932, 2010

16. Allton K, Jain AK, Herz HM, Tsai WW, Jung SY, Qin J, Bergmann A, Johnson RL and Barton MC: Trim24 targets endogenous p53 for degradation. Proc Natl Acad Sci USA 106: 11612-11616, 2009.

17. Jain AK and Barton MC: Regulation of p53: TRIM24 enters the RING. Cell Cycle 8: 3668-3674, 2009.

18. Livak KJ and Schmittgen TD: Analysis of relative gene expression data using real-time quantitative PCR and the 2(-Delta Delta C(T)) method. Methods 25: 402-408, 2001.

19. Jiang S, Minter LC, Stratton SA, Yang P, Abbas HA, Akdemir ZC, Pant V, Post S, Gagea M, Lee RG, et al: TRIM24 suppresses development of spontaneous hepatic lipid accumulation and hepatocellular carcinoma in mice. J Hepatol 62: 371-379, 2015. 
20. Li H, Sun L, Tang Z, Fu L, Xu Y, Li Z, Luo W, Qiu X and Wang E: Overexpression of TRIM24 correlates with tumor progression in non-small cell lung cancer. PLoS One 7: e37657, 2012.

21. Debatin KM: Apoptosis pathways in cancer and cancer therapy. Cancer Immunol Immunother 53: 153-159, 2004.

22. Stennicke HR and Salvesen GS: Biochemical characteristics of caspases-3,-6,-7, and-8. J Biol Chem 272: 25719-25723, 1997.

23. Lavrik IN, Golks A and Krammer PH: Caspases: Pharmacological manipulation of cell death. J Clin Invest 115: 2665-2672, 2005.

24. Hong B, Lui VW, Hashiguchi M, Hui EP and Chan AT: Targeting tumor hypoxia in nasopharyngeal carcinoma. Head Neck 35 : $133-145,2013$

25. Nyberg P, Salo T and Kalluri R: Tumor microenvironment and angiogenesis. Front Biosci 13: 6537-6553, 2008.

26. Bai Y, Zhu X, Chao J, Zhang Y, Qian C, Li P, Liu D, Han B, Zhao L, Zhang J, et al: Pericytes contribute to the disruption of the cerebral endothelial barrier via increasing VEGF expression: Implications for stroke. PloS one 10: e0124362, 2015.

27. Des Guetz G, Uzzan B, Nicolas P, Cucherat M, Morere JF, Benamouzig R, Breau JL and Perret GY: Microvessel density and VEGF expression are prognostic factors in colorectal cancer Meta-analysis of the literature. Br J Cancer 94: 1823-1832, 2006.

28. Alvarez JV, Greulich H, Sellers WR, Meyerson M and Frank DA: Signal transducer and activator of transcription 3 is required for the oncogenic effects of non-small-cell lung cancer-associated mutations of the epidermal growth factor receptor. Cancer Res 66: 3162-3168, 2006.
29. Buettner R, Mora LB and Jove R: Activated STAT signaling in human tumors provides novel molecular targets for therapeutic intervention. Clin Cancer Res 8: 945-954, 2002.

30. Mullighan CG, Zhang J, Harvey RC, Collins-Underwood JR, Schulman BA, Phillips LA, Tasian SK, Loh ML, Su X, Liu W, et al: JAK mutations in high-risk childhood acute lymphoblastic leukemia. Proc Natl Acad Sci USA 106: 9414-9418, 2009.

31. Grandis JR, Drenning SD, Zeng Q, Watkins SC, Melhem MF, Endo S, Johnson DE, Huang L, He Y and Kim JD: Constitutive activation of Stat 3 signaling abrogates apoptosis in squamous cell carcinogenesis in vivo. Proc Natl Acad Sci 97: 4227-4232, 2000.

32. Xiong $\mathrm{H}$, Zhang ZG, Tian XQ, Sun DF, Liang QC, Zhang YJ, Lu R, Chen YX and Fang JY: Inhibition of JAK1, 2/STAT3 signaling induces apoptosis, cell cycle arrest, and reduces tumor cell invasion in colorectal cancer cells. Neoplasia 10: 287-297, 2008.

33. Du W, Hong J, Wang YC, Zhang YJ, Wang P, Su WY, Lin YW, Lu R, Zou WP, Xiong H and Fang JY: Inhibition of JAK2/STAT3 signalling induces colorectal cancer cell apoptosis via mitochondrial pathway. J Cell Mol Med 16: 1878-1888, 2012.

34. Lv D, Li Y, Zhang W, Alvarez AA, Song L, Tang J, Gao WQ, $\mathrm{Hu}$ B, Cheng SY and Feng H: TRIM24 is an oncogenic transcriptional co-activator of STAT3 in glioblastoma. Nat Commun 8: $1454,2017$. 